\title{
Spacecraft Orbit Determination and Its Control Strategy
}

Haiyan Xuan ${ }^{1, a}$, Leibo Song ${ }^{2, b}$, Peiliang $\mathrm{Hao}^{3, \mathrm{c}}$, Hang $\mathrm{Ji}^{4, \mathrm{~d}}$ and Xianwei $\mathrm{Li}^{5, \mathrm{e}}$

${ }^{1}$ School of Economics and Management, Lanzhou University of Technology, Lanzhou 730050, China;

${ }^{2}$ School of science, Lanzhou University of Technology, Lanzhou 730050, China;

${ }^{3}$ College of Electrical and Information Engineering, Lanzhou University of Technology, Lanzhou 730050, China;

${ }^{4}$ College of Petrochemical Engineering, Lanzhou University of Technology, Lanzhou 730050, China;

${ }^{5}$ School of Mechanical \& Electronical Engineering, Lanzhou University of Technology, Lanzhou 730050, China.

\author{
ahaiyanxuan@msn.com, bleibosong.lut@gmail.com, \\ c574669397@qq.com, ${ }^{\mathrm{d}}$ 13919935093@163.com, ${ }^{\mathrm{e}}$ 398812053@qq.com
}

Keywords: Orbit determination, soft landing, dynamics, control strategy.

\begin{abstract}
In this paper, we got the aircraft soft landing trajectory model based on the dynamic model, at the same time, regard saving energy and reducing the economic costs as the main purpose, respectively, we gave the corresponding optimal control strategy for each orbit phase.
\end{abstract}

\section{Introduction}

In the condition of determining the orbit, the optimal control is very important, which can not only reduce costs, but also can save the energy, optimization control theory method has different kinds, good method will provide a theoretical basis for the aviation sector. In order to identify practical methods orbit model, many researchers at home and abroad for a soft way orbit carried out a large number of model studies. Jain and others proposed a method based on multi-resolution to solve the trajectory optimization problems[1]. Wang and others completed the study that applying the genetic algorithm in the soft landing trajectories optimization under the condition with constant thrust amplitude[2]. Bonami and others consider the integer and continuous variables for a plane trajectory optimization method[3]. Peng and others studied rapid orbit optimization problem about fixed point soft landing of lunar lander by using Gauss pseudo method[4]. Applying elasticity of rope, complex state and the influence of control and constraint, under the rope system, Liu and others studied the optimal control problem of the orbit transfer in satellite surface[5]. Garg proposed a optimal trajectory control problem directly at the point of the LGR(Legendre-Gauss-Radau)[6]. In this paper, based on the knowledge of dynamics, method of determining aircraft landing trajectory is given, and Based on the principle of optimization, researching a parameter control method combining dynamics to solve the spacecraft precise fixed point soft landing optimal control problem, Then, obtaining the soft landing optimal orbit and realizing resource conservation.

\section{Aircraft Soft Landing Trajectory Model}

It's very important to determine aircraft soft landing orbit for Precision landing in space, dynamic orbit determination is a basic method for precise orbit determination of spacecraft. Here, we first give the orbit model, specific methods are as follows:

The equations of motion detectors in the month fixed coordinate can be expressed as:

$$
\begin{gathered}
\dot{x}_{l}=V_{x l}, \quad \dot{y}_{l}=V_{y l}, \dot{y}_{l}=V_{y l}, m=-F / C \\
V_{x l}=O F / m-g_{x l}+2 \omega_{l} \\
V_{y l}=P F / m-g_{y l}
\end{gathered}
$$




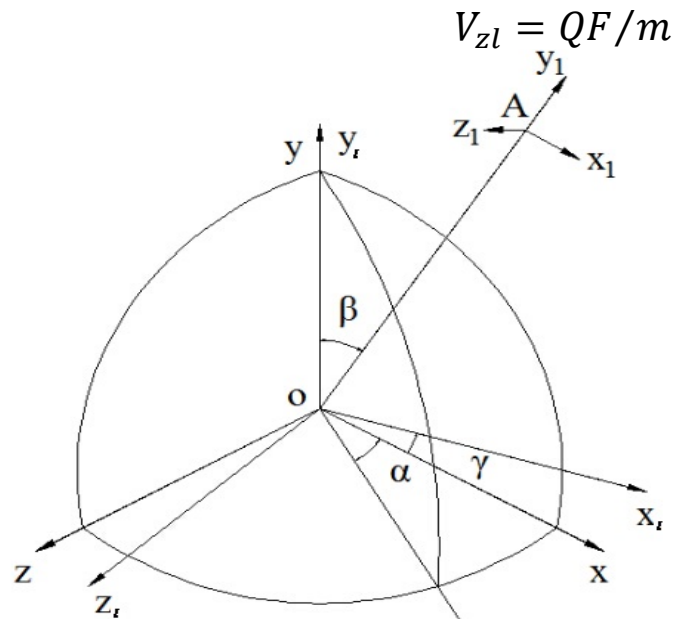

Fig. 1 The first coordinate system

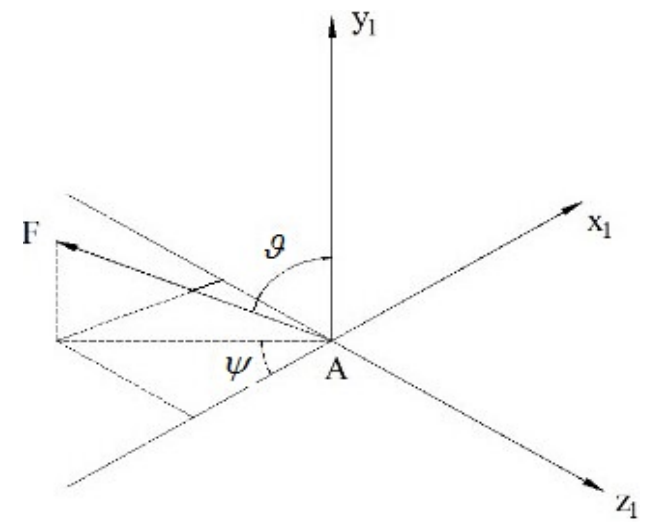

Fig. 2 The second coordinate system

In Fig. 1, we define the inertial coordinate system oxyz, the origin in the month, the reference plane is lunar equatorial plane, ox axis directs the ascending node of the moon's equator relative to moons path, oy axis directs the moon rotation direction, oz axis is determined by the right rule. Again, we define the monthly fixed coordinate system $\mathrm{ox}_{\mathrm{l}} \mathrm{y}_{\mathrm{l}} \mathrm{z}_{\mathrm{l}}$, the reference plane is the moon equatorial plane, The $\mathrm{ox}_{\mathrm{l}}$ axis points to the line of intersection of the equatorial plane and the initial meridian plane, oy $\mathrm{y}_{1}$ axis directs the moon rotation direction, $\mathrm{oz}_{\mathbf{l}}$ axis is determined by the right rule.

In Fig. 2, $A x_{1} y_{1} z_{1}$ is the orbit coordinate system that the origin is the center of the aircraft, $A y_{1}$ points to the direction from the moon's heart to the extension line of aircraft, $\mathrm{Ax}_{1}$ points to direction of movement and $A x_{1}$ perpendicular to the $A y_{1}, A z_{1}$ axis is determined by the right rule.

Here:

$$
\begin{aligned}
O= & (\cos \alpha \cos \beta \cos \gamma-\sin \alpha \sin \gamma) \sin \vartheta \cos \psi-(\sin \alpha \cos \beta \cos \gamma+\cos \alpha \sin \gamma) \sin \vartheta \sin \psi \\
& +\sin \beta \cos \gamma \cos \vartheta \\
P= & \cos \alpha \sin \beta \sin \vartheta \cos \psi+\cos \beta \cos \vartheta+\sin \alpha \sin \beta \sin \vartheta \sin \psi \\
Q= & (\cos \alpha \cos \beta \sin \gamma-\sin \alpha \cos \gamma) \sin \vartheta \cos \psi-(\sin \alpha \cos \beta \sin \gamma+\cos \alpha \cos \gamma) \sin \vartheta \sin \psi \\
& +\sin \beta \sin \gamma \cos \vartheta \\
G_{x l}= & \frac{G_{M}}{x_{l}^{2}+y_{l}^{2}+z_{l}^{2}}+\frac{x_{l}}{\sqrt{x_{l}^{2}+y_{l}^{2}+z_{l}^{2}}}
\end{aligned}
$$

By using $\mathrm{y}_{\mathrm{l}}, \mathrm{z}_{\mathrm{l}}$ instead of $\mathrm{x}_{\mathrm{l}}$, we can obtain $\mathrm{g}_{\mathrm{xl}}, \mathrm{g}_{\mathrm{yl}}, \mathrm{G}_{\mathrm{M}}$ is moon gravitational constant. $\mathrm{C}$ is a

braking rockets specific impulse, it's a constant, $\mathrm{m}$ is the quality of aircraft, $\mathrm{F}$ is the engine thrust.

Boundary conditions:

The initial condition for the lunar lander with dimensionless:

$$
\left\{\begin{array}{l}
\dot{v}_{r 0}=0 \\
\dot{v}_{\theta 0}=\frac{v_{\theta 0}}{\sqrt{R_{L} / G_{M}}} \\
\dot{r}_{0}=\frac{R_{L}+h_{0}}{R_{L}} \\
\theta_{0}=\theta
\end{array}\right.
$$

Here: $v_{\theta 0}$ is The initial speed of the decline stage, $h_{0}$ is the initial height of dropping, generally it's $15 \mathrm{~km}$.

The terminal condition for the lunar lander with dimensionless:

$$
\left\{\begin{array}{l}
\dot{\mathrm{v}}_{\mathrm{rf}}=0 \\
\dot{\mathrm{v}}_{\theta \mathrm{f}}=\frac{\mathrm{v}_{\theta 0}}{\sqrt{\mathrm{R}_{\mathrm{L}} / \mathrm{G}_{\mathrm{M}}}} \\
\dot{\mathrm{r}}_{\mathrm{f}}=\frac{\mathrm{R}_{\mathrm{L}}+\mathrm{h}_{\mathrm{f}}}{\mathrm{R}_{\mathrm{L}}} \\
\theta_{\mathrm{f}}=\theta+\Delta \theta
\end{array}\right.
$$


$h_{f}$ is the aircraft height at the end of decline stage, generally it's $2 \mathrm{~km} . R_{L}$ is the radius of the moon, $\theta$ is polar angle of aircraft.

\section{Optimal Control Strategy}

Based on the principle of optimal fuel consumption, using the parameter control, we converted dynamic optimal control problem for a static parameter optimization problem, by numerical calculation, and then get the detector's optimal trajectory. Specific methods are as follows:

Take $\left[\mathrm{x}_{1}, \mathrm{y}_{\mathrm{l}}, \mathrm{z}_{\mathrm{l}}, \mathrm{V}_{\mathrm{xl}}, \mathrm{V}_{\mathrm{yl}}, \mathrm{V}_{\mathrm{zl}}, \mathrm{m}\right]^{\prime}$ as the system state variables, and we regard $\mathrm{u}=[\vartheta, \psi, \mathrm{F}]^{\prime}$ as the control variable.

Orbital coordinate system to the inertial coordinate system transformation matrix:

$$
T_{1}=\left[\begin{array}{ccc}
\cos \alpha \cos \beta & \sin \beta & -\sin \alpha \cos \beta \\
-\cos \alpha \sin \beta & \cos \beta & \sin \alpha \sin \beta \\
\sin \alpha & 0 & \cos \alpha
\end{array}\right]
$$

Inertial coordinate system to the fixed coordinate system transformation matrix:

$$
T_{2}=\left[\begin{array}{ccc}
\cos \gamma & 0 & -\sin \gamma \\
0 & 1 & 0 \\
\sin \gamma & 0 & \cos \gamma
\end{array}\right]
$$

1) The main reduction stage, fuel consumption optimal control strategy

According to the requirements of the fuel consumption optimal, take performance indicators for this:

In addition there are constraints:

$$
J=k \sqrt{V_{x l}^{2}\left(t_{f}\right)+V_{y l}^{2}\left(t_{f}\right)+V_{z l}^{2}\left(t_{f}\right)}+C^{-1} \int_{0}^{t_{f}} F d t
$$

$$
G=\left\{\begin{array}{l}
g_{1}=x_{l}\left(t_{f}\right)-x_{l f}=0 \\
g_{2}=y_{l}\left(t_{f}\right)-y_{l f}=0 \\
g_{3}=z_{l}\left(t_{f}\right)-z_{l f}=0 \\
g_{3}=\sqrt{x_{l}^{2}+y_{l}^{2}+z_{l}^{2}}-r_{f} \geq 0
\end{array}\right.
$$

Here:

$x_{l f}, y_{l f}, z_{l f}$ as scheduled landing site coordinates in month fixed coordinate system, and landing site to month heart distance, the radius of the moon is $r_{f}=\sqrt{x_{l f}^{2}+y_{l f}^{2}+z_{l f}^{2}}$.

In the system of (1), (2), (3), (4) to satisfy the constraint function under the condition of G, obtain the suitably controlled variable $u$ to make index (7) reaches the minimum.

2) Rapid adjustment stage, time optimal control strategy

Rapid adjustment stage, in order to land smoothly, the aircraft mainly to adjust their position, time optimization is the mainly optimization strategy on the stage.

3) Obstacle avoidance stage, line optimal control strategy

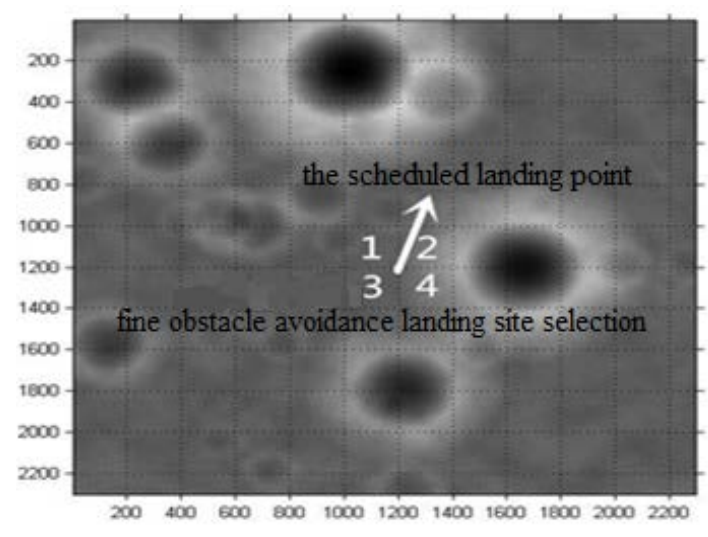

Fig. 3 coarse obstacle avoidance control strategy

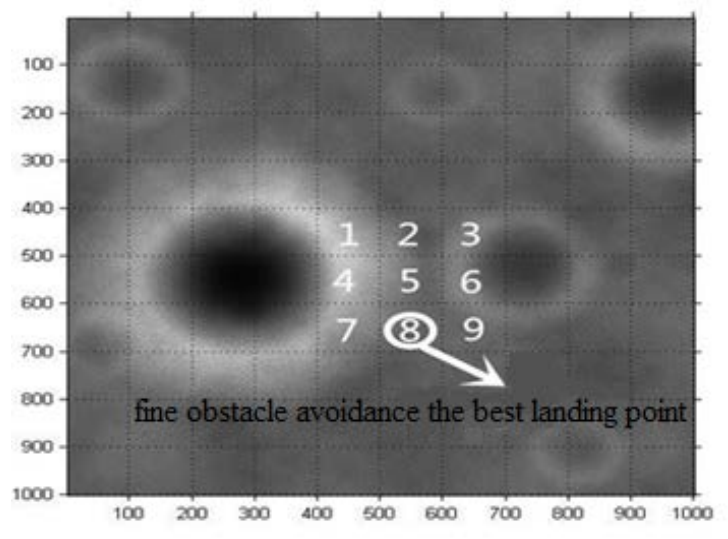

Fig. 4 fine obstacle avoidance control strategy 
Coarse obstacle avoidance area: at 2300*2300: the gray value of the image by reading 2300 height, the grey value is stored in a matrix of $2300 * 2300$; after establishing a $200 * 200$ matrix that it all element is 1 ,ach point on the $2300 * 2300$ pixel area will be scanned, and to make it gray variance,

and through the comparison of variance each scanning area, take the minimum for optimal landing area.

Run the program using MATLAB, by two layers of loop will conform to the conditions of scanned grey value area all into 1 , is not in conformity will be value of 0 ; In the end, the grey value of all area to be 1 is the optimal landing area.

Because it is the essence of obstacle avoidance, it should be fixed matrix size reduction, reduceng $1000 * 1000$ to $100 * 100$, later in the same way to scan the areas of pixel can be $1000 * 1000$, in order to better reflect the degree of the smooth surface of the landing, the optimal landing area is the gray variance minimum.(as shown in Fig. 3 and Fig. 4)

4) Decline stage with slow speed

This stage to control the aircraft uniform descent, then, the moon's attractiveness and reducing engine thrust balance each other. By the definition of specific impulse and the law of conservation of momentum:

$$
\int_{0}^{t} v_{e} d t=\Delta m v
$$

here: $\mathrm{m}_{1} \mathrm{v}_{1}-\mathrm{m}_{2} \mathrm{v}_{2}=\mathrm{v}_{\mathrm{e}} \Delta \mathrm{m}$, The deceleration stage propellant consumption for $\Delta \mathrm{m}, \mathrm{v}_{\varepsilon}$ is specific impulse, then, we should make deceleration motor thrust equal to the aircraft 's gravity.

5) free fall stage

Because the aircraft is still free fall, so here we don't do optimal control.

\section{Summary}

Dynamic orbit determination is a basic method for precise orbit determination of spacecraft. This paper presents the corresponding theory of dynamic orbit determination, in the case of determining orbit, we also focus on the corresponding period optimization problem in orbit determination condition by adding the parameter. With the rapid development of the aerospace technology at domestic and foreign, measurement and control requirements and conditions become more and more diversified, many new problems began to appear as energy conservation and economic project and so on, it needs to find a more suitable method to deal with different situations.

\section{References}

[1] S. Jain, P. Tsiotras, Trajectory optimization using multiresolution techniques, Journal of Guidance, Control, and Dynamics. 31 (2008) 1424-1436.

[2] Wang Jie, Li Jun-feng, Cui nai-gang, Liu Dun, Genetic algorithm optimization of lunar probe soft-landing trajectories, Journal of Tsinghua University, 43 (2003) 1056-1059.

[3] P. Bonami, A. Olivares, M. Soler, E. Staffetti, Multiphase Mixed-Integer Optimal Control Approach to Aircraft Trajectory Optimization, Journal of Guidance, Control, and Dynamics. 36 (2013) 1267-1277.

[4] Peng Qi-bo, Li Hai-yang, Shen Hong-xin, Rapid Lunar Exact-Landing Trajectory Optimization Via Gauss Pseudospectral Method, Jounml of Astronautics, 31 (2010) 1012-1016.

[5] Liu Li-li, Wen Hao, Jin Dong-ping, Hu Hai-yan, Optimal Control of Orbit Transfer for a Tethered Subsatellite System, Acta aeronautica et astronautica sinica, 30 (2009) 332-336.

[6] D. Garg, M.A. Patterson, C. Francolin, C.L. Darby, G.T. Huntington, W.W. Hager, A.V. Rao, Direct trajectory optimization and costate estimation of finite-horizon and infinite-horizon optimal control problems using a Radau pseudospectral method, Computational optimization and applications. 49 (2011) 335-358. 